\title{
Star Counts and Spectral Classification in Star Formation Regions of the Magellanic Clouds
}

\section{Kontizas}

University of Athens, Panepistimiopolis, Athens 15783, Greece

D. H. Morgan

Royal Observatory, Blackford Hill, Edinburgh EHg 3HJ, UK

Y. Bellas-Vellidis, E. Kontizas

National Observatory of Athens, Athens 11810, Greece

\begin{abstract}
Large complexes of young objects are common features in galaxies and are called superassociations, complexes and supercomplexes according to their size. They contain HII regions, early-type stars, associations and young clusters. Such large structures are expected to trace the star formation regions within galaxies. Star counts and spectral classification over extended areas, using direct and objective prism Schmidt telescope plates are good methods of determining the boundaries of these complexes and supercomplexes where the spatial density of early-type stars is enhanced. A project of tracing the supercomplexes in the Magellanic Clouds has started using plates taken with the UK $1.2 \mathrm{~m}$ Schmidt Telescope.
\end{abstract}

\section{Introduction}

The Large Magellanic Cloud (LMC) is known to be an irregular galaxy; but it has strong similarities to a spiral galaxy in that there are extensive, active, star formation regions, though in the LMC these lack clear symmetry. We do not know to what extent this asymmetry is related to the LMC's original formation and/or to its encounter with our Galaxy (and the SMC), though it is probably true that the LMC has been affected by this event through the triggering of star and star cluster formation during the past $10^{8}$ years. Efremov (1988), using the LMC and 30 Dor in particular as prime examples, has summarized star formation in terms of the hierarchical clustering of stellar groupings: associations, groups of a few associations (called aggregates), larger regions called complexes which consist of three or more groupings of associations, bright emission nebulae and/or aggregates, and finally supercomplexes which consist of at least two complexes. Complexes and supercomplexes must also have young stars distributed between their dominant components.

Feitsinger \& Braunsworth (1984) have identified levels of clustering with typical sizes of 100,400 and $1500 \mathrm{pc}$. Martin et al. (1984) have also noted the 
existence of these groupings from similarities between the radial velocities of the supergiant stars in them and the nearest HI complex. Fifty-two HI complexes have been identified by McGee \& Milton (1966) with internal velocity dispersions of $\sim 11 \mathrm{~km} / \mathrm{s}$ which are similar to those found in the groupings of supergiant stars. Also, studies of Cepheids (Isserstedt 1984) and star cluster ages (Hodge 1973 ) have shown the existence of huge groupings of $\sim 1.3 \mathrm{kpc}$ in size. According to Efremov (1988) all this demonstrates a discrete star formation process which is large-scale in both space and time.

\section{Programme}

Stellar complexes and star formation regions do not contain just hot, bright stars, gas, dust and protostars; they also have a more populous fainter component belonging to the main sequence which may prove to be a better indicator of their sizes and densities. It is therefore important to search for this fainter component which, if present, will be revealed by a higher number density of stars than in the surrounding regions of the parent galaxy. The isopleths of the fainter stellar components in and around star formation regions will allow us to determine the shapes and dimensions of these structures. The existence of earlytype stars throughout these high density regions will be a necessary criterion for deciding whether we have identified a stellar complex. Finally, stellar radial velocities measured over large areas can provide a third means of defining these systems.

This paper describes the start of a large project designed to make detailed studies of the complexes and supercomplexes in the LMC. Schmidt telescopes with their wide fields-of-view are ideal tools for this type of work; we shall use photographic plates taken with the UK $1.2 \mathrm{~m}$ Schmidt Telescope (UKST). The deepest sky survey plates taken with the UKST would allow the detection of main sequence stars down to a spectral type of $\sim \mathrm{G} 0$; but these plates are too crowded for this project. We have decided to start the project using plates taken in the U-waveband; these typically reach $\mathrm{m} \sim 20-21$ and provide an excellent compromise between depth and crowding. Moreover, the blue stars of the upper main sequence stand out against the older, background, stellar populations more clearly on these plates than on plates taken in redder wavebands. These direct plates will be measured and star counts will be analysed in terms of large-scale structures. Parallel spectroscopy of stars found within these structures will be carried out using objective prism plates taken through the $2400 \AA / \mathrm{mm}$ dispersion objective prism in the $3300-5500 \AA$ waveband. This classification can be carried out to \pm 1 spectral class down to $\mathrm{m}_{B} \sim 18.5$.

\section{Progress}

In preliminary work (Maravelias et al. 1992, Kontizas et al. 1994), we made star counts in and around some of the well-known LMC constellations (with sizes up to $1^{\circ}$ ) and tried to set useful criteria for determining their dimensions. First, we looked for those areas which had star number densities more than 1,2 or $3 \sigma$ above the mean number density in the area in question where $\sigma$ is the rms scatter of those densities. (The area measured included both the constellation 


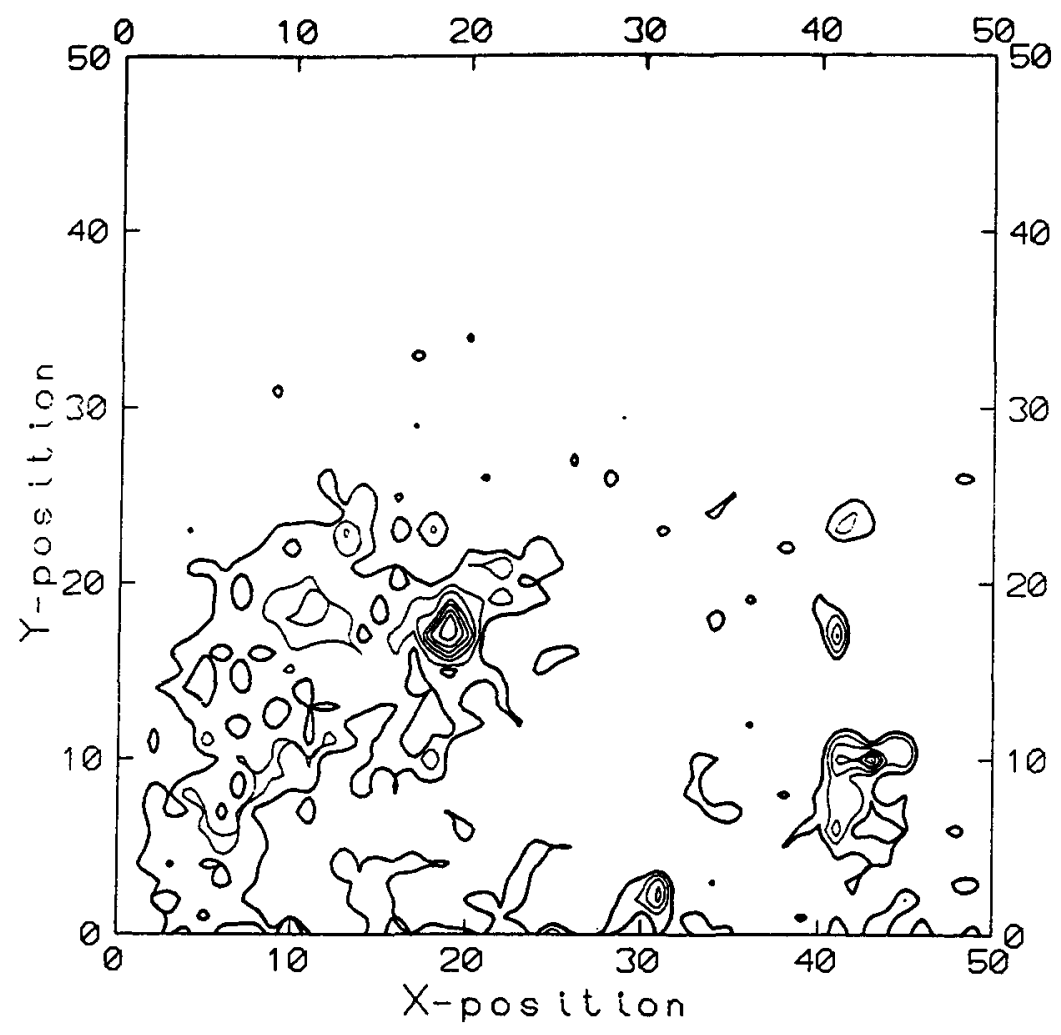

Figure 1. Isopleths within a $2^{\circ} \times 2^{\circ}$ area of the LMC seen on UKST plate U10576. The centre of the area is $5 \mathrm{~h} 9 \mathrm{~m},-65^{\circ} 17^{\prime}(1950)$; the data were obtained using the COSMOS measuring machine and analysed using the software package GRAPHER. The minimum isopleth shown is $3 \sigma$ above the mean stellar density (see text) and the other isopleths are in units of $\sigma$. The association LH43 is at $(\mathrm{X}, \mathrm{Y})=(20,20)$. 
and a significant background region surrounding it.) It was found that isopleths 1 or $2 \sigma$ above the mean showed a general gradient across the area in question but without revaling any closed structures. However, isopleths at $3 \sigma$ above the mean did reveal large structures which were found through subsequent objective prism classification work to contain early-type stars, nebulae and associations. These results were found in four constellations from measurements made by eye.

A substantial survey of the LMC has now started using the fast measuring machines COSMOS and APM. Fig 1 shows isopleths $3 \sigma$ and greater above the mean stellar density within a $2^{\circ} \times 2^{\circ}$ region measured with COSMOS from plate U10576. In this figure, there is one strong concentration of stars which is identified as the association LH43 (Lucke \& Hodge 1970) with diameter $\sim 80 \mathrm{pc}$, and several other small groupings of stars all contained within a complex of diameter $\sim 500 \mathrm{pc}$. More sophisticated techniques can be used to fit the background isopleths against which the complexes are seen. It will also be necessary to look at areas larger than $\sim 1^{\circ}$ to see whether more extensive structures are present. The next stage will be to study the spectral types of the stars in this region and thereby confirm whether the complex is indeed an extensive, young, stellar system. The technique will then be applied to other regions of the LMC.

\section{Acknowledgements}

The authors would like to thank the staff of the UK Schmidt Telescope Unit for providing the excellent plates and the staffs of the COSMOS and APM groups at ROE and RGO for providing the measurements.

\section{References}

Efremov Y. N., 1988, Ap. Sp. Sci. Rev., 7, 105

Feitsinger J. V. \& Braunsfurth E., 1984, A\&A, 139, 104

Hodge P. W., 1973, AJ, 78, 807

Isserstedt J., 1984, A\&A, 41, 21

Kontizas E., Maravelias S. E., Dapergolas A., Bellas-Vellidis Y. \& Kontizas M., 1994, in Astronomy from Wide-Field Imaging, Proc. IAU Symp. 161, in press

Lucke P. B. \& Hodge P. W., 1970, AJ, 75, 171

Maravelias S. E., Kontizas E., Kontizas M. \& Dapergolas A., 1992, in Working Group in Wide-Field Astronomy Newsletter, 3, 12

Martin N., Maurice E., Prévot L., Rebeirot E. \& Rousseau J., 1984, in Structure and Evolution of the Magellanic Clouds, Proc. IAU Symp. 108, S. van den Bergh \& K. S. de Boer, eds, p.137

McGee R. X. \& Milton J. A., 1966, Austr. J. Phys., 19, 343 